\title{
Inhomogeneous HRV Spectral Complexity: a Preliminary Evaluation With Gravitational Stimuli Under Selective Autonomic Blockade
}

\author{
Juan Bolea ${ }^{1}$, Gaetano Valenza ${ }^{2}$, Raquel Bailón ${ }^{1}$, Esther Pueyo ${ }^{1}$, Riccardo Barbieri ${ }^{3}$ \\ ${ }^{1}$ Biomedical Signal Interpretation and Computational Simulation Group (BSICoS), CIBER-BBN, \\ University of Zaragoza, Zaragoza, Spain \\ ${ }^{2}$ Bioengineering and Robotics Research Center E. Piaggio \& Department of Information \\ Engineering, University of Pisa, Pisa, Italy \\ ${ }^{3}$ Department of Electronics, Informatics and Bioengineering, Politecnico di Milano, Milano, Italy
}

\begin{abstract}
Nonlinear Heart Rate Variability (HRV) analysis has been successfully applied to assess autonomic nervous system (ANS) regulation of the cardiovascular system in health and disease. Here, the spectral complexity framework is introduced for characterization of time-varying $H R V$ spectral components.

Correlation dimension, $D_{2}$, and maximum approximate entropy $A p E n_{\text {max }}$, were calculated on time-varying $H R V$ spectral series derived from a linear point-process model, which was fitted to RR series from 14 healthy subjects undergoing selective ANS blockade during postural changes.

Complexity quantification of the instantaneous LF component using $D_{2}$ revealed significantly higher values in standing vs. supine under atropine (vagal blockade), but not under propranolol (sympathetic blockade), in contrast to the results found under control conditions. Similar trends were found for ApEn $n_{\max }$. Conversely, no statistically significant differences were found in the complexity indices evaluated for the instantaneous HF component, either for standing vs. supine or blockades vs. control.

The proposed framework enriches the current knowledge on complex ANS regulation of the heart, supporting the fact that previously reported reduction in cardiovascular complexity during postural changes is mainly vagally driven.
\end{abstract}

\section{Introduction}

Cardiovascular and respiratory dynamics are regulated by autonomic nervous system (ANS) activity to maintain a stable homeostatic status. Nonlinear interaction between the sympathetic and parasympathetic branches of ANS, which is responsible for heart rate regulation, have been well established [1]. Heart rate variability (HRV) series, resulting from this interaction, exhibits complex behavior [2-4].
HRV analysis has been proven as a promising noninvasive technique to assess ANS activity [2]. Besides quantification of heartbeat linear dynamics through HRV spectra, nonlinear techniques and related complexity estimates have allowed effective discrimination between physiological and pathological conditions, such as hypertension, diabetes, myocardial infarction, depression, and congestive heart failure $[2,3]$.

To retrieve instantaneous HRV estimates defined in the time and frequency domains, an inhomogeneous pointprocess modeling approach, where ANS modulation of heartbeat is represented by Inverse-Gaussian (IG) probability density functions predicting the occurrence of $\mathrm{R}$-waves in the electrocardiogram, can be applied [5,6]. The instantaneous heart rate may then be parametrized in a linear autoregressive function characterizing the first-order moment of such a continuous IG distribution.

Extensions of this framework to the nonlinear domain including instantaneous high-order spectra, instantaneous entropy and second-order complexity characterization, i.e. complexity variability, have been recently investigated (see [4] and references therein). However, the intrinsic complex dynamics inherent to instantaneous HRV spectra have not been considered yet.

To this end, in this study we introduce a complexity analysis applied onto series derived from instantaneous spectra of linear point-process models. Particularly, correlation dimension and maximum approximate entropy are computed on point-process-derived instantaneous mean and standard deviation of heartbeat as well as on instantaneous spectral series obtained by integrating the instantaneous spectra within the low frequency (LF) and highfrequency (HF) bands $(0.04-015$ and $0.15-0.4 \mathrm{~Hz}$, respectively). Note that series of instantaneous LF may be referred as a marker of sympatho-vagal dynamics, whereas series of instantaneous HF may be referred as a marker of vagal dynamics [7]. We test the proposed methodology in heartbeat series from 14 healthy subjects who were administered pharmacological ANS blockers and performed a protocol of body postural changes. 


\section{Materials and Methods}

\subsection{Experimental Data}

Pharmacological ANS blockades database (HMS-MITFFMS): The database consists of 78 ECG signals, 7minute duration each, recorded at $360 \mathrm{~Hz}$ on 13 male healthy subjects with no history of cardiopulmonary disease (age range 19-38 y.o.). Subjects were informed and gave their written consent in accordance with the Massachusetts Institute of Technology Committee on the Use of Humans as Experimental Subjects before each procedure.

First, ECGs were recorded while subjects were in supine position control condition (SUC), i.e. no drug was administered. Then, subjects were changed to standing position (STC) and ECGs were recorded when hemodynamic equilibrium was reached. In a second stage, subjects were divided into two groups. One group consisted of 7 subjects $(20.29 \pm 1.25$ y.o.) who were administered atropine (0.03 $\mathrm{mg} / \mathrm{kg})$ and the other one of 6 subjects $(23 \pm 6.5$ y.o. $)$ who were administered propranolol $(0.2 \mathrm{mg} / \mathrm{kg})$. ECGs were recorded in supine and standing positions when hemodynamic equilibrium was reached (SUA, STA, SUP, and STP respectively). Although a third stage was included where drug administration was exchanged between the groups, this stage was discarded and only control and single blockades were considered for this study. Further details of this database can be found elsewhere [8].

\subsection{Methodology of Signal Processing}

Pre-processing: R-waves were identified from the ECG by a wavelet-based QRS detector. The obtained RR interval series were checked and eventually corrected to be free of ectopic beats and algorithmic artifacts using a pointprocess based algorithm [9].

Point-process modeling: Point-process models provide a probabilistic framework to characterize the stochastic properties of beat-to-beat variations through an inverse Gaussian probability distribution [5]:

$$
\begin{aligned}
f\left(t \mid H_{t(k), \theta}\right) & =\left[\frac{\theta_{p+1}}{2 \pi(t-t(k))^{3}}\right]^{\frac{1}{2}} . \\
& \exp \left(-\frac{\theta_{p+1}\left(t-t(k)-\mu\left(H_{t(k)}, \theta\right)\right)^{2}}{2 \mu\left(H_{t(k)}, \theta\right)^{2}(\mathbf{t}-\mathbf{t}(\mathbf{k}))}\right)
\end{aligned}
$$

where $t(k)$ represents the time occurrence of the $k^{t h}$ heartbeat, $H_{t(k)}=\{t(k), w(k), w(k-1), \ldots, w(k-p+1)\}$ the elements involved in the history-dependence of $p$ order of previous beats $(p=12)$ and $w(k)=t(k)-t(k-1)$ the RR values.

The instantaneous mean RR was computed as:

$$
\mu_{R R(t)}=\mu\left(H_{t(k), \theta}\right)=\theta_{0}+\sum_{j=1}^{p} \theta_{j} w(k-j+1)
$$

The estimation of the $\theta$ parameters was performed by considering a local maximum-likelihood estimation within a time window $l(l=60 \mathrm{~s})$ [5]. Kolmogorov-Smirnov test was used to measure the goodness-of-fit of heartbeat events $t(k)$ and those of the point-process distributions after time-rescaling application [5]. The parameters $\theta$ were updated by shifting the estimation interval $\Delta \mathrm{ms}$, i.e. the time resolution (in our case $50 \mathrm{~ms}$ ).

The instantaneous standard devision was estimated as follows: $\quad \sigma_{R R(t)}=\left[\mu\left(H_{t(k), \theta}\right)^{3} \theta_{p+1}^{-1}\right]^{\frac{1}{2}}$

Point-process time-varying spectral time series: The parametric description provided in Eq.1 allows for the extraction of time-varying spectral components. The instantaneous power spectra was computed as follows:

$$
S_{R R(f, t)}=\frac{\sigma_{R R(t)}^{2}}{\left|1-\sum_{i=1}^{p} \theta_{i}(t) z^{-i}\right|_{z=e^{j 2 \pi f}}}
$$

Low frequency power $\left(P_{L F}: 0.04-0.15 \mathrm{~Hz}\right)$ and high frequency power $\left(P_{H F}: 0.15-0.45 \mathrm{~Hz}\right)$ content were instantaneously estimated. Moreover, normalized $P_{L F}(t)$, computed as $P_{L F n}(t)=P_{L F}(t) /\left(P_{L F}(t)+P_{H F}(t)\right)$, was considered for further analysis.

Time domain indices: Heart rate mean (HRM) and the root mean square of standard deviation (RMSSD) of $\mu_{R R(t)}$ were computed.

Nonlinear indices: Correlation dimension, $D_{2}$, and maximum approximate entropy, $\operatorname{ApEn}\left(2, r_{\max }(2)\right)$, hereinafter denoted by $A p E n_{\max }$ for the sake of simplification, were considered as related to the complexity and irregularity of the time series [10]. These nonlinear indices were computed from point-process derived time series: instantaneous mean $\mu_{R R(t)}$; instantaneous standard deviation $\sigma_{R R(t)}$; and time-varying power spectral components. The employed notation was as follows: $X^{Y}$, where $X \in\left\{D_{2}, A p E n_{\max }\right\}$, and $Y \in$ $\left\{\mu_{R R(t)}, \sigma_{R R(t)}, P_{L F}(t), P_{H F}(t), P_{L F n}(t)\right\}$. Of note, the embedding dimension $m$ values were adjusted to the time series resolution. Consequently, reported values of $m$ when time resolution was $500 \mathrm{~ms}$ were multiplied by a factor of 10 at $50 \mathrm{~ms}$.

Statistical analysis: The HMS-MIT-FFMS database was analyzed by considering the last 5 minutes of each ECG recording at each of the body postural phases and considered conditions (control and pharmacological ANS blockades). Paired t-test or Mann-Whitney U test were applied according to the K-S test results. A $p$-value $<0.05$ was considered for statistical significance.

\section{Results}

The results from the complexity analysis of the $\mu_{R R(t)}$ series (see Fig. 1a)) showed statistically significant differences between supine and standing for the two complexity indices only when analyzing control conditions. A statistically significant decrease was also found in complexity values under atropine versus control in supine position. Similarly, a significant reduction was found under atropine with respect to control in standing position for $A p E n_{\max }$ index 
a)

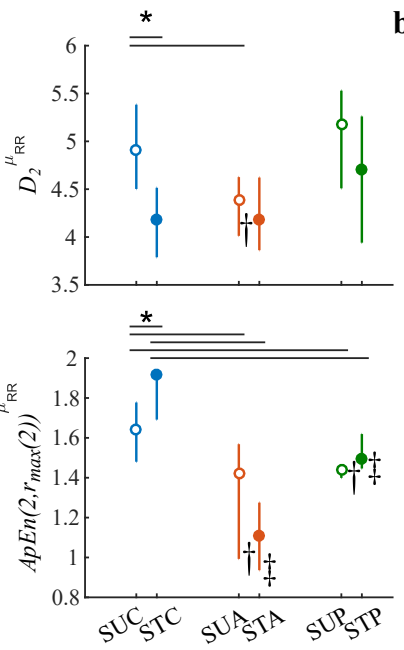

b)

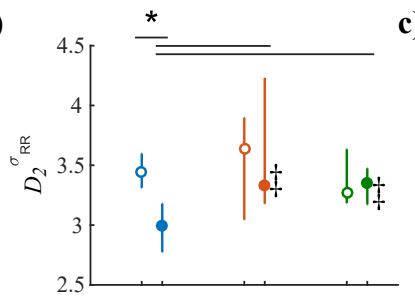

c)

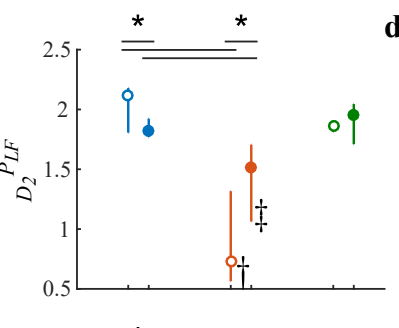

d)

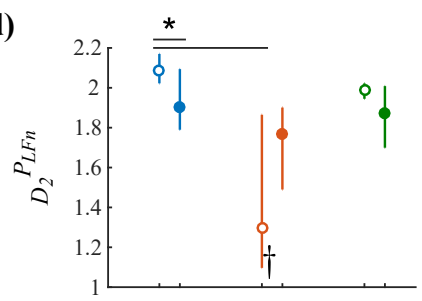

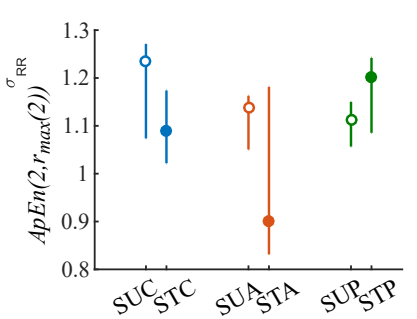
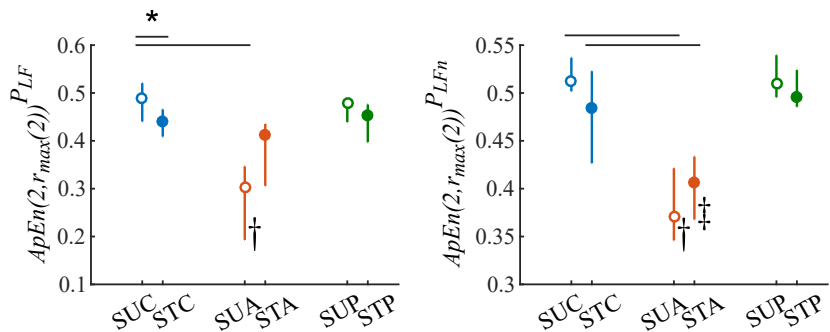

Figure 1. $\quad D_{2}$ and $A p E n_{\max }$ computed over: a) instantaneous RR $\left(\mu_{R R(t)}\right)$; b) instantaneous RR variations $\left(\sigma_{R R(t)}\right)$; c) and d) time-varying spectral time series $P_{L F}(t)$ and $P_{L F n}(t)$ evaluating the effect of ANS blockades. $*$ represents statistical significant differences between SUX and STX, where $X \in\{C$ (control); $A$ (atropine); $P$ (propranolol) $\}$. $\dagger$ SUA, SUP vs. SUC, and $\ddagger$ STA, STP vs. STC. $p$-value $<0.05$ is considered as significant by Mann-Whitney U test.

\begin{tabular}{lcccccc}
\hline & SUC & STC & SUA & STA & SUP & STP \\
\hline$D_{2}{ }^{\mu_{R R(t)}}$ & $4.9(4.5 \mid 5.4)$ & $4.2(3.8 \mid 4.5) *$ & $4.4(4 \mid 4.6) \dagger$ & $4.2(3.9 \mid 4.6)$ & $5.2(4.5 \mid 5.5)$ & $4.7(3.9 \mid 5.3) *$ \\
$A p E n_{\max } \mu_{R R(t)}$ & $1.6(1.5 \mid 1.8)$ & $1.9(1.7 \mid 1.9) *$ & $1.4(0.99 \mid 1.6) \dagger$ & $1.1(0.94 \mid 1.3) \ddagger$ & $1.4(1.4 \mid 1.5) \dagger$ & $1.5(1.4 \mid 1.6) * \ddagger$ \\
$D_{2}{ }^{\sigma}{ }_{R(t)}$ & $3.4(3.3 \mid 3.6)$ & $3(2.8 \mid 3.2) *$ & $3.6(3 \mid 3.9)$ & $3.3(3.2 \mid 4.2) \ddagger$ & $3.3(3.2 \mid 3.6)$ & $3.4(3.2 \mid 3.5) * \ddagger$ \\
$A p E n_{\max }{ }^{\sigma}{ }_{R R(t)}$ & $1.2(1.1 \mid 1.3)$ & $1.1(1 \mid 1.2)$ & $1.1(1.1 \mid 1.2)$ & $0.9(0.83 \mid 1.2)$ & $1.1(1.1 \mid 1.1)$ & $1.2(1.1 \mid 1.2)$ \\
$D_{2} P_{L F}(t)$ & $2.1(1.8 \mid 2.2)$ & $1.8(1.8 \mid 1.9) *$ & $0.73(0.56 \mid 1.3) \dagger$ & $1.5(1.1 \mid 1.7) * \ddagger$ & $1.9(1.8 \mid 1.9)$ & $2(1.7 \mid 2)$ \\
$A p E n_{\max } P_{L F}(t)$ & $0.49(0.44 \mid 0.52)$ & $0.44(0.41 \mid 0.47) *$ & $0.3(0.19 \mid 0.35) \dagger$ & $0.41(0.31 \mid 0.44)$ & $0.48(0.44 \mid 0.49)$ & $0.45(0.4 \mid 0.48)$ \\
$D_{2} P_{L F n}(t)$ & $2.1(2 \mid 2.2)$ & $1.9(1.8 \mid 2.1) *$ & $1.3(1.1 \mid 1.9) \dagger$ & $1.8(1.5 \mid 1.9)$ & $2(1.9 \mid 2)$ & $1.9(1.7 \mid 2)$ \\
$A p E n_{\max } P_{L F n}(t)$ & $0.51(0.5 \mid 0.54)$ & $0.48(0.43 \mid 0.52)$ & $0.37(0.35 \mid 0.42) \dagger$ & $0.41(0.37 \mid 0.43) \ddagger$ & $0.51(0.5 \mid 0.54)$ & $0.5(0.49 \mid 0.52)$ \\
\hline
\end{tabular}

Table 1. $D_{2}$ and $A p E n_{\max }$ computed over: instantaneous RR $\left(\mu_{R R(t)}\right)$; instantaneous RR variations $\left(\sigma_{R R(t)}\right)$; and timevarying spectral time series $P_{L F}(t)$; and $P_{L F n}(t)$ evaluating the effect of ANS blockades. $*$ represents statistical significant differences between SUX and STX, where $X \in\{C$ (control); $A$ (atropine); $P$ (propranolol) $\}$. $†$ SUA, SUP vs. SUC, and $\ddagger$ STA, STP vs. STC. $p$-value $<0.05$ is considered as significant by Mann-Whitney $\mathrm{U}$ test.

but $D_{2}$, since this value was already low in the standing control stage. Nonlinear indices revealed no changes regarding body positions after administration of propranolol.

The analysis of $\sigma_{R R(t)}$ series (see Fig. 1b)) resulted in a decrease in complexity indices, although only significant for $D_{2}$ in standing with respect to supine position in control. No significant differences were found between supine and standing under atropine or propranolol. However, $D_{2}$ was found significantly higher under any of the two blockades with respect to control.

Complexity analysis on $P_{H F}(t)$ series did not reveal any statistical difference either at control or under ANS blockades.

On the other hand, complexity analysis of instantaneous LF power revealed significant changes between body positions during control conditions. Although both indices showed changes in the comparison of supine and standing under atropine, these were only statistically different for $D_{2}^{P_{L F}(t)}$ (Fig. $\left.1 \mathrm{~d}\right)$ ). In addition, $P_{L F n}(t)$ analysis (see Fig. 1e)) showed a decrease in the complexity values when comparing standing and supine at control and under propranolol, whereas the effect of atropine administration was associated with an increase in their mean distribution value. Complexity values were statistically lower under atropine as compared to control.

\section{Discussion and Conclusion}

We proposed a methodology to investigate ANS nonlinear dynamics through complexity analysis of instantaneous spectral indices derived from inhomogeneous pointprocess models. To this extent, we computed complexity and irregularity indices such as $D_{2}$ and $A p E n_{\max }$ on instantaneous LF and HF series during selective ANS blockades in healthy subjects undergoing postural changes. Particularly, the protocol comprised simple gravitational stimuli obtained by transitioning from supine to standing position. 
Our results suggest that atropine, used to inhibit parasympathetic activity on cardiovascular control, mostly affected the irregularity and complexity of point-process derived series. Subjects under parasympathetic blockade showed a significant reduction in $D_{2}$ and $A p E n_{\max }$ values with respect to control conditions. Conversely, the effect of propranolol, used to inhibit sympathetic activity, was not reflected in complexity changes. The results obtained by analyzing instantaneous RR time series were found to be in accordance with previous studies [11]

Regarding the instantaneous RR variance, $\sigma_{R R}(t)$, our results suggest that lower complexity of sympatho-vagal dynamics is associated with upright position with respect to supine position at control, as revealed through $D_{2}$.

The statistical differences found in supine versus standing under control conditions disappeared following administration of any of the two ANS blockers, which highlights the contribution of both the sympathetic and parasympathetic branches to the observed postural-induced changes in the analyzed indices.

However, significantly greater values of $D_{2}$ and $A p E n_{\max }$ were found when drugs were administered during standing position with respect to control stage. Blockades produced a variation in the ANS modulation tone which is also manifested in the complexity values.

The novelty of this study lies in the complexity and irregularity analysis of HRV spectral components extracted from instantaneous heartbeat estimates defined within a point process framework. In fact, although these spectral components are usually assumed to be stationary, as well as their nonlinear/complex behavior, our analysis shows a decrease of $P_{L F n}(t)$ complexity induced by atropine when compared to control conditions. The regulation of the parasympathetic nervous system is highly reduced by atropine, thus ANS modulation is mainly due to the sympathetic modulation. The elicitation of ANS by postural change was captured by indices evaluating the complexity and irregularity of $P_{L F}(t)$. Once parasympathetic activity was reduced by the blockade, the increase of sympathetic activity was expressed as an increment of complexity of $P_{L F}(t)$. When sympathetic activity was inhibited by propranolol, ANS changes due to body positions were not captured by complexity analysis and the analysis results were similar to the ones found in control conditions.

In conclusion, the proposed methodological framework enriches current knowledge on complex ANS regulation of the heart, supporting previously reported, vagally-driven reductions in cardiovascular complexity.

\section{Acknowledgements}

This work was funded under projects TIN2014-53567-R and DPI2016-75458-R by MINECO (Spain) and by BSICOS Group (T96) from Government of Aragn and European Social Fund (EU) and by the European Research Council (ERC) through project ERC-2014-StG 638284. CIBER is a center of the Instituto de Salud Carlos III in assistance from the European Regional Development Fund.
The computation was performed by the ICTS "NANBIOSIS", more specifically by the High Performance Computing Unit of the CIBER in Bioengineering, Biomaterials \& Nanomedicine (CIBER-BBN) at the University of Zaragoza.

\section{References}

[1] Sunagawa K, Kawada T, Nakahara T. Dynamic nonlinear vago-sympathetic interaction in regulating heart rate. Heart Vessels 1998;13:157-174.

[2] Task Force of the ESC-NASPE. Heart rate variability: Standards of measurement, physiological interpretation, and clinical use. Circulation 1996;93(1):1043-1065. ISSN 15612775

[3] Sassi R, et al. Advances in heart rate variability signal analysis: Joint position statement by the e-Cardiology ESC Working Group and the European Heart Rhythm Association co-endorsed by the Asia Pacific Heart Rhythm Society. Europace sep 2015;17(9):1341-1353. ISSN 15322092.

[4] Valenza G, et al. Complexity Variability Assessment of Nonlinear Time-Varying Cardiovascular Control. Scientific reports feb 2017;7:42779. ISSN 2045-2322.

[5] Barbieri R, et al. A point-process model of human heartbeat intervals: new definitions of heart rate and heart rate variability. AJP Heart and Circulatory Physiology sep 2004; 288(1):H424-H435. ISSN 0363-6135.

[6] Bailón R, et al. The integral pulse frequency modulation model with time-varying threshold: application to heart rate variability analysis during exercise stress testing. EEE Trans Biomed Eng 2011;58(3):642-652.

[7] Valenza G, Citi L, Saul JP, Barbieri R. Measures of sympathetic and parasympathetic autonomic outflow from heartbeat dynamics. Journal of Applied Physiology 2018;.

[8] Sobh JF, et al. Database for ECG, arterial blood pressure, and respiration signal analysis: feature extraction, spectral estimation, and parameter quantification. In Proc. of the Ann. Int. Conf. IEEE Engineering Medical Biology Society. 1995; 955-956.

[9] Citi L, Brown EN, Barbieri R. A real-time automated pointprocess method for the detection and correction of erroneous and ectopic heartbeats. IEEE Trans Biomed Eng oct 2012;59(10):2828-37. ISSN 1558-2531.

[10] Bolea J, et al. Methodological framework for estimating the correlation dimension in HRV signals. Computational and Mathematical Methods in Medicine 2014;2014.

[11] Bolea J, et al. Non-linear HRV indices under autonomic nervous system blockade. Proceedings of the Annual International Conference of the IEEE Engineering in Medicine and Biology Society EMBS 2014;2014:3252-3255.

Address for correspondence:

Juan Bolea

Edif. I+D+i Mariano Esquillor S/N Zaragoza Spain

jbolea@unizar.es 